\title{
Moments and Identities Involving Inverted Wishart Distribution
}

\author{
RAJA MOHAMMAD LATIF \\ Department of Mathematics and Natural Sciences \\ Prince Mohammad Bin Fahd University \\ P.O. Box 1664 Al Khobar \\ KINGDOM OF SAUDI ARABIA

\begin{abstract}
ANWAR H. JOARDER
Faculty of Science and Engineering

Northern University of Business and Technology Khulna BANGLADESH
\end{abstract}

\begin{abstract}
Moments of multivariate Wishart Distribution are known up to fourth order. But in many contexts, moments of some functions of Wishart distribution and Inverted Wishart Distribution have been found useful in risk theoretic estimation of covariance matrix and its characteristics. In this paper we review moments of some important functions of Wishart and Inverted Distributions.
\end{abstract}

Key-Words: - Wishert distribution, Inverted Wishert distribution, Covariance matrix, Wishert matrix, Jacobian, Moments

Received: November 13, 2019. Revised: February 24, 2020. Accepted: April 17, 2020. Published: April $29,2020$.

\section{Introduction}

The inverted Wishart distribution has got various applications in statistics. For instance, the distribution can be used as a natural conjugate prior when dealing with Bayesian estimation of covariance matrix under sampling from multivariate normal distribution (Anderson, 2003, Section 7.7). Moments of the inverted Wishart distribution have also been utilized in discriminant analysis (Das Gupta, 1968; Siskind, 1972 and Haff, 1982) while obtaining moments of the maximum likelihood estimators in the growth curve model (von Rosen, 1988 and Von Rosen, 1997). Useful results for the inverted Wishart distribution can also be found in Press (1982). Kaufman (1967) derived the moments by factorization theorem. Das Gupta (1968) utilized some invariance arguments. Both of them based their calculations on the inverse moments of chisquare variable. In a series of papers, Haff (1977, $1979,1980,1981,1982)$ presented moment identities which are useful for deriving moments of inverted Wishart distribution. The identities were established by applying Stokes' theorem. Independently of Haff, von Rosen (1985) derived moments of inverted Wishart distribution with the help of matrix calculus. These moments have been found useful in risk theoretic estimation of covariance matrix and its characteristics. See for example Joarder (1997) and Joarder (1998). Let $X_{1}, X_{2}, \cdots X_{N}(N>p) \quad$ be a $p$-dimensional independent normal random vector with mean vector $\bar{X}$ so that the sums of squares and cross product matrix is given by $\sum_{j=1}^{N}\left(X_{j}-\bar{X}\right)\left(X_{j}-\bar{X}\right)^{\prime}=A$.

Fisher (1915) derived the distribution of $A$ for $p=2$ in order to study the distribution of correlation coefficient from a normal sample. Wishart (1928) obtained the distribution for arbitrary $p$ as the joint distribution of sample variances and covariances from multivariate normal population. Because of its important role in multivariate statistical analysis, various authors have derived it from different perspectives. See the references in Gupta and Nagar (2000, 87-88). The 
following theorem provides the density function of Wishart matrix.

Theorem 1.1 The random symmetric positive definite matrix $A$ is said to have a Wishart distribution with parameters $p, m=N-1>p$ and $\Sigma(p \times p)>0$, written as $A \square W_{p}(m, \Sigma) \quad$ if its probability density function is given by

$$
\begin{aligned}
& f_{1}(A)=c_{p, m}|\Sigma|^{-m / 2}|A|^{(m-p-1) / 2} \exp \left(-\frac{1}{2} t r \Sigma^{-1} A\right), \\
& A>0, m>p
\end{aligned}
$$

where

$$
c_{p, m}^{-1}=2^{m p / 2} \Gamma_{p}(m / 2)
$$

and

$$
\Gamma_{p}(\alpha)=\pi^{p(p-1) / 4} \prod_{i=1}^{p} \Gamma(\alpha-(i-1) / 2), .
$$

$\alpha>(p-1) / 2$

(See e.g. Anderson, 2003, 252).

It is worth mentioning that $a_{11} / \sigma_{11} \square \chi_{m}^{2}$. The first moment of $A, \operatorname{tr} A, \operatorname{det}(A), A^{-1}$ and similar beautiful quantities are known (Muirhead, 1982). Some moments of functions of $p \times p$ Wishart matrix are provided below:

(i) $E(A)=m \Sigma$,

(ii) $E\left(A^{\square}\right)=N \Sigma, A^{\square}=\sum_{j=1}^{N}\left(X_{j}-\mu\right)\left(X_{j}-\mu\right)^{\prime}$,

$\mu$ known,

(iii) $(m-p-1) E\left(A^{-1}\right)=\Sigma^{-1}$,

(iv) $E\left(|A|^{k}\right)=\frac{2^{k p} \prod_{i=1}^{p} \Gamma\left(\frac{m+2 k}{2}-\frac{i-1}{2}\right)}{\prod_{i=1}^{p} \Gamma\left(\frac{m}{2}-\frac{i-1}{2}\right)}|\Sigma|^{k}$
(Voinov and Nikulin, 1996, 197-198). A nice update of moments of Wishart distribution is given in Gupta and Nagar (2000). By differentiating the moment generating function of $A \square W_{p}(m, \Sigma)$, $m>p$, de Waal and Nel (1973) derived the following results:

$$
\begin{aligned}
& (a) E\left(A^{2}\right)=m\left((m+1) \Sigma+(t r \Sigma) I_{p}\right) \Sigma \\
& (b) E\left(A^{3}\right)=m\left(\begin{array}{l}
\left(m^{2}+3 m+4\right) \Sigma^{2}+2(m+1)(t r \Sigma) \Sigma \\
+(m+1)\left(t r \Sigma^{2}\right) I_{p}+(t r \Sigma)^{2} I_{p}
\end{array}\right) \Sigma .
\end{aligned}
$$

De Waal and Nel (1973) also derived the fourth moment of Wishart matrix. But in many contexts, moments of some functions of Wishart distribution have been found useful. In this paper we review some moments of functions of Wishart Distribution and also derive expectation of some functions of Inverted Wishart Distribution along Muirhead (1986).

\section{Moments and Identities Involving Wishart Matrix}

(v) $E(\operatorname{tr} A)=m \operatorname{tr} \sum$, he following theorem and corollary are due to (vi ) $E\left(t r A^{\square}\right)=m$ tr $\sum$, $\mu$ known,

(ix) $E\left[(m+1)(\operatorname{tr} A)^{2}-2 \operatorname{tr}\left(A^{2}\right)\right]=$ (vii ) $E\left[(\text { trA })^{2}\right]=m^{\text {Theqreph }}+2 m^{2}$ Suppese) that $A \sim W_{p}(m, \Sigma)$ defined $(m-1) m(m+2) \operatorname{tr}\left(\Sigma^{2}\right)$,

(x) $E\left[(m+2)\left(t r A^{\square}\right)^{2}-2 \operatorname{tr}\left(A^{\llbracket 2}\right)\right]=$

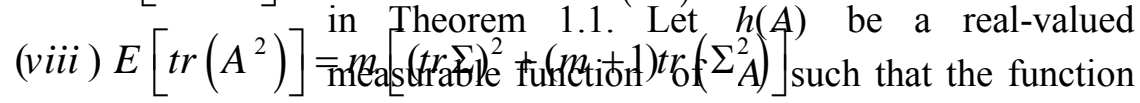
$f(t ; A)=h(t A), t>0, \quad$ is differentiable at $t=1$. $m(m+1)(m+3) \operatorname{tr}\left(\Sigma^{2}\right), \mu$ known, Again let $f^{\prime}(t ; A)=\frac{\partial}{\partial t} f(t ; A)$. Then

(xi) $E\left[m \operatorname{tr}\left(A^{2}\right)-(\operatorname{tr} A)^{2}\right]=$ $(m-1) m(m+2) \operatorname{tr}\left(\Sigma^{2}\right)$,

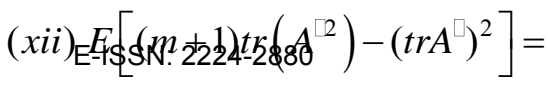




$$
E\left[\operatorname{tr}\left(\Sigma^{-1} A\right) h(A)\right]=m p E[h(A)]-2 E\left[f^{\prime}(1 ; A)\right]
$$

Corollary 2.1 Let $h(x A)=x^{l} h(A)$ for some $l$.

Then $E\left[\operatorname{tr}\left(\Sigma^{-1} A\right) h(A)\right]=(m p+2 l) h(A)$.

For any nonnegative integer $k$ we will be using the notation:

$a_{\{k\}}=a(a+1)(a+2) \cdots(a+k-1)$,

$a^{\{k\}}=a(a-1)(a-2) \cdots(a-k+1)$.

That is $a_{\{k\}}=(a+k-1)^{\{k\}}, a^{\{k\}}=(a-k+1)^{\{k\}}$.

If $A \sim W_{p}(m, \Sigma)$, then the following results are known.

(i) $E\left(|A|^{k}\right)=\frac{2^{p k} \Gamma_{p}(m / 2+k)}{\Gamma_{p}(m / 2)}|\Sigma|^{k}$,

(ii) $E\left(A^{-1}\right)=\frac{1}{m-p-1} \Sigma^{-1}, m>p+1$

The following moments and identities derived by Corollary 2.1 are due to Muirhead (1986).

1. $E\left\lfloor\left(\operatorname{tr} \Sigma^{-1} A\right)^{k}\right\rfloor=2^{k}(m p / 2)_{\{k\}}$.

2. $E\left[\left(\operatorname{tr} \Sigma^{-1} A\right)^{-k}\right]=\frac{2^{-k}}{(m p / 2-1)^{\{k\}}}, m p>2 k$.

3. $E\left\lfloor\left(t r \Sigma^{-1} A\right)^{k} \operatorname{tr}(A)\right\rfloor=2^{k} m(m p / 2+1)_{\{k\}}(\operatorname{tr} \Sigma)$

4. $E\left[\left(\operatorname{tr} \Sigma^{-1} A\right)^{k} \operatorname{tr}\left(A^{-1}\right)\right]=$

$\frac{2^{k}(m p / 2-1)_{\{k\}}}{m-p-1} \operatorname{tr} \Sigma^{-1}, m>p+1$

5. $E\left[\left(\operatorname{tr} \Sigma^{-1} A\right)^{k} \operatorname{tr}\left(\Sigma A^{-1}\right)\right\rfloor=$

$2^{k} \frac{m(m p / 2-1)_{\{k\}}}{m-p-1}, m>p+1$
6. $E\left[\left(t r \Sigma^{-1} A\right)^{k}|A|^{h}\right]=$

$2^{h p+k}(m p / 2+h p)_{\{k\}} \frac{\Gamma_{p}(m / 2+h)}{\Gamma_{p}(m / 2)}|\Sigma|^{h}$

7. $E\left[\left(t r \Sigma^{-1} A\right)(\operatorname{tr} A)\left(\operatorname{tr} A^{\alpha}\right)\right]=$

$[m p+2(\alpha+1)] E\left[(\operatorname{trA})\left(t r A^{\alpha}\right)\right]$

Putting $\Sigma=I$ we have the identity

$E\left[(t r A)^{2}\left(t r A^{\alpha}\right)\right]=$

$[m p+2(\alpha+1)] E\left[(\operatorname{tr} A)\left(t r A^{\alpha}\right)\right]$

which is the Sharma and Krishnamoorthy (1984) Identity.

\section{Main Results}

The probability distribution function of the inverted Wishart matrix $B=A^{-1}$ is given in the following theorem.

Theorem 1.1 Suppose that the random symmetric positive definite matrix $A$ has the Wishart distribution written as $A \sim W_{p}(m, \Sigma)$ with parameters $p, m=N-1>p$ and $\Sigma(p \times p)>0$. Then $B=A^{-1}$ has the probability density function $f(B)=$

$c_{p, m}|\Psi|^{m / 2}|B|^{-(m+p+1) / 2} \exp \left(-\frac{1}{2} \operatorname{tr} \Psi B^{-1}\right)$,

$B>0, m>p$,

where

$c_{p, m}^{-1}=2^{m p / 2} \Gamma_{p}(m / 2)$,

$\Gamma_{p}(\alpha)=$

$\pi^{p(p-1) / 4} \prod_{i=1}^{p} \Gamma(\alpha-(i-1) / 2), \alpha>(p-1) / 2$, and

$\Psi=\Sigma^{-1}$.

The density function in (1.1) will be denoted by $B \sim W_{p}^{-1}(\Psi, m)$. See e.g. Andeson, 2003, 272) or Anderson $(1984,268)$. 
Proof. The Jacobian of the transformation $A=B^{-1}$ is given by $J(A \rightarrow B)=|B|^{-(p+1)}$ (See Gupta and Nagar, 200, 14). Then it follows from Theorem 1.1

$$
f_{1}(A)=c_{p, m}|\Sigma|^{-m / 2}|A|^{(m-p-1) / 2} \exp \left(-\frac{1}{2} \operatorname{tr} \Sigma^{-1} A\right)
$$

That

$$
\begin{aligned}
& f(B)=c_{p, m}\left|\Psi^{-1}\right|^{-m / 2}\left|B^{-1}\right|^{(m-p-1) / 2} \\
& \exp \left(-\frac{1}{2} \operatorname{tr} \Psi B^{-1}\right)|B|^{-(p+1)}
\end{aligned}
$$

which simplifies to

$$
\begin{aligned}
& f(B)= \\
& c_{p, m}|\Psi|^{m / 2}|B|^{-(m+p+1) / 2} \exp \left(-\frac{1}{2} \operatorname{tr} \Psi B^{-1}\right), \\
& \text { where } \quad c_{p, m}^{-1}=2^{m p / 2} \Gamma_{p}(m / 2), \\
& \Gamma_{p}(\alpha)= \\
& \pi^{p(p-1) / 4} \prod_{i=1}^{p} \Gamma(\alpha-(i-1) / 2), \alpha>(p-1) / 2
\end{aligned}
$$

and $\Psi=\Sigma^{-1}$.

If $p=1$, then the corresponding univariate density function would be

$$
f_{3}\left(b_{11}\right)=\frac{1}{2^{m / 2} \Gamma(m / 2)} \psi_{11}^{m / 2} b_{11}^{-(m+1+1) / 2} \exp \left(-\frac{\psi_{11}}{2 b_{11}}\right),
$$

so that the density function of

$V=\psi_{11} / b_{11}$ is given by

$$
\begin{aligned}
& f_{4}(v)= \\
& \frac{1}{2^{m / 2} \Gamma(m / 2)} \psi_{11}^{m / 2}\left(\frac{\psi_{11}}{v}\right)^{-(m+2) / 2} e^{-v / 2}\left(\frac{\psi_{11}}{v^{2}}\right), \\
& f_{4}(v)=\frac{1}{2^{m / 2} \Gamma(m / 2)} v^{(m / 2)-1} e^{-v / 2},
\end{aligned}
$$

It is worth mentioning that $U=b_{11} / \psi_{11}>0$ has an inverted chi-square distribution with density function

$$
f_{5}(u)=\frac{u^{-(m / 2)-1} e^{-1 /(2 u)}}{2^{m / 2} \Gamma(m / 2)}, u>0
$$

$$
\text { i.e. } 1 / U \sim \chi_{m}^{2} \text {. }
$$

\section{Lemma 3.1}
(i) $E\left(|B|^{h}\right)=\frac{|\Psi|^{h} \Gamma_{p}(m / 2-h)}{2^{h p} \Gamma_{p}(m / 2)}, m / 2>h$
(ii) $E(B)=\frac{1}{m-p-1} \Psi, m>p+1$

\section{Proof.}

(i) $E\left(|B|^{h}\right)=$

$\int_{B>0}|B|^{h} c_{p, m}|\Psi|^{m / 2}|B|^{-(m+p+1) / 2}$

$\exp \left(-\frac{1}{2} \operatorname{tr} \Psi B^{-1}\right) d B$

$=\left(c_{p, m}|\Psi|^{m / 2}\right) \int_{B>0}|B|^{h}|B|^{-(m-2 h+p+1) / 2}$

$\exp \left(-\frac{1}{2} \operatorname{tr} \Psi B^{-1}\right) d B$

$=\left(c_{p, m}|\Psi|^{m / 2}\right) \frac{1}{c_{p, m-2 h}|\Psi|^{(m-2 h) / 2}}$

The moment then follows by (1.1).

(ii) See Anderson $(2003,273)$ or Mardia, Kent and Bibby $(1979,85)$

\section{Main Results}

Theorem 4.1 Suppose that $B \sim I W_{p}(\Psi, m)$ defined in Theorem 1.1. Let $h(B)$ be a real-valued measurable function of $B$ such that the function $f(t ; B)=h(t B), t>0$, is differentiable at $t=1$. Again let $f^{\prime}(t ; B)=\frac{\partial}{\partial t} f(t: B)$.

Then

$$
E\left[\operatorname{tr}\left(\Psi B^{-1}\right) h(B)\right]=m p E[h(B)]-2 E\left[f^{\prime}(1 ; B)\right] \text {. }
$$

Proof. For $t>0$, define the function $g(t)$ as 


$$
\begin{aligned}
& g(t)= \\
& c_{p, m}|\Psi|^{m / 2} t^{m p / 2} \int_{B>0} h(B)|B|^{-(m+p+1)} e^{-\frac{t}{2} t r \Psi B^{-1}} d B
\end{aligned}
$$

where $\quad c_{p, m}=2^{m p / 2} \Gamma_{p}(m / 2)$ and note that $g(1)=E[h(B)]$. Rewrite

$g(t)=c_{p, m}|\Psi|^{m / 2} \int_{B>0} h(B)|B|^{-(m+p+1)} t^{m p / 2} e^{-\frac{t}{2} t r \Psi B^{-1}} d B$

Differentiating with respect to $t$ (justified by dominated convergence provided $E\left[\operatorname{tr}\left(\Psi B^{-1}\right) h(B)\right]$ exist $)$, we have

$g^{\prime}(t)=c_{p, m}|\Psi|^{m / 2} \int_{B>0} h(B)|B|^{-(m+p+1)}$

$\left[\frac{m p}{2} t^{(m p-2) / 2} e^{-\frac{t}{2} t r \Psi B^{-1}}+t^{m p / 2} e^{-\frac{t}{2} t r \Psi B^{-1}}\left(-\frac{1}{2} t r \Psi B^{-1}\right)\right] d B$

or,

$$
\begin{aligned}
& g^{\prime}(t)=c_{p, m}|\Psi|^{m / 2} \int_{B>0} h(B)|B|^{-(m+p+1)} \\
& {\left[\frac{m p}{2} t^{(m p-2) / 2}+t^{m p / 2}\left(-\frac{1}{2} \operatorname{tr} \Psi B^{-1}\right)\right] e^{-\frac{t}{2} t r \Psi B^{-1}} d B}
\end{aligned}
$$

Then

$$
\begin{gathered}
g^{\prime}(1)=c_{p, m}|\Psi|^{m / 2} \int_{B>0} h(B)|B|^{-(m+p+1)} \\
{\left[\frac{m p}{2}+\left(-\frac{1}{2} \operatorname{tr} \Psi B^{-1}\right)\right] e^{-\frac{1}{2} t r \Psi B^{-1}} d B} \\
\text { or, } g^{\prime}(1)=\frac{m p}{2} E[h(B)]-\frac{1}{2} E\left[\operatorname{tr}\left(\Psi B^{-1}\right) h(B)\right] .
\end{gathered}
$$

Now make the transformation $B=t Y^{-1}$ in (2.2) with Jacobian $J\left(B \rightarrow Y^{-1}\right)=t^{p(p+1) / 2}$ since $B$ is a symmetric matrix (equation 2.15 .7 of Press, 1982) so that

$$
\begin{aligned}
& g(t)=c_{p, m}|\Psi|^{m / 2} \int_{Y^{-1}>0} h\left(t Y^{-1}\right)\left|t Y^{-1}\right|^{-(m+p+1) / 2} t^{m p / 2} \\
& e^{-\frac{t}{2} t r \Psi t^{-1} Y}\left[t^{p(p+1) / 2} d Y^{-1}\right] \\
& =c_{p, m}|\Psi|^{m / 2} \int_{Y^{-1}>0} f\left(t: Y^{-1}\right)|Y|^{(m+p+1) / 2} e^{-\frac{t}{2} t r \Psi t^{-1} Y} d Y^{-1}
\end{aligned}
$$

and then differentiating we have

$$
\begin{aligned}
& g^{\prime}(t)= \\
& c_{p, m}|\Psi|^{m / 2} \int_{Y^{-1}>0} f^{\prime}\left(t: Y^{-1}\right)|Y|^{(m+p+1) / 2} e^{-\frac{t}{2} t r \Psi t^{-1} Y} d Y^{-1}
\end{aligned}
$$

Since $Y^{-1}=B$ for $t=1$, we have

$$
\begin{aligned}
& \left.g^{\prime}(1)=c_{p, m}|\Psi|^{m / 2} \int_{Y^{-1}>0}|B|^{-(m+p+1) / 2} f^{\prime}(1: B)\right) \\
& e^{-\frac{1}{2} t r \Psi Y} d Y^{-1}
\end{aligned}
$$

i.e. $g^{\prime}(1)=E\left[f^{\prime}(1 ; B)\right]$

The identity in (4.1) follows from (4.3) and (4.4). In many applications, the function $h($.$) has the$ property that, for $u>0, h(u B)=u^{l} h(B)$ for some real $l$. Then $f(t: B)=h(t B)=t^{l} h(B)$, so that $f^{\prime}(1: B)=l h(B)$. Then we have the following corollary.

Corollary 4.1: Let $h(x B)=x^{l} h(B)$ for some $l$. Then

$$
E\left\lfloor\operatorname{tr}\left(\Psi B^{-1}\right) h(B)\right\rfloor=(m p-2 l) h(B)
$$

By the use of the above corollary and Lemma 1.1 we have derived the moments of some useful functions of inverted Wishart matrix $B$ in the next section.

\section{Some Special Moments}


1. $E\left[\left(\operatorname{tr}\left(\Psi B^{-1}\right)\right)^{k}\right]=2^{k}(m p / 2)_{\{k\}}$.

Proof. Let $E\left\lfloor\left(\operatorname{tr}\left(\Psi B^{-1}\right)\right)^{k}\right\rfloor=E\left[\operatorname{tr}\left(\Psi B^{-1}\right) h_{1}(B)\right]$ where $\quad h_{1}(B)=\left(\operatorname{tr}\left(\Psi B^{-1}\right)\right)^{k-1} \cdot \quad$ Then $h_{1}(x B)=\left(\operatorname{tr}\left(\Psi(x B)^{-1}\right)\right)^{k-1}=x^{-k+1} h_{1}(B)$ so that by Corollary 2.1, we have $l=-(k-1)$ and $E\left[\operatorname{tr}\left(\Psi B^{-1}\right) h_{1}(B)\right]=(m p-2 l) E\left[h_{1}(B)\right]$, i.e. $E\left\lfloor\left(\operatorname{tr}\left(\Psi B^{-1}\right)\right)^{k}\right\rfloor=$ $[m p+2(k-1)] E\left[\left(\operatorname{tr}\left(\Psi B^{-1}\right)\right)^{k-1}\right]$.

Next, let $E\left[\left(\operatorname{tr}\left(\Psi B^{-1}\right)\right)^{k-1}\right]=E\left[\operatorname{tr}\left(\Psi B^{-1}\right) h_{2}(B)\right]$ where $h_{2}(B)=\left(\operatorname{tr}\left(\Psi B^{-1}\right)\right)^{k-2}$.

Then $h_{2}(x B)=x^{-k+2} h_{2}(B)$ so that by Corollary 2.1, we have $\quad l=-(k-2) \quad$ and $E\left[\operatorname{tr}\left(\Psi B^{-1}\right) h_{2}(B)\right]=(m p-2 l) E\left[h_{2}(B)\right]$, i.e $E\left[\left(\operatorname{tr}\left(\Psi B^{-1}\right)\right)^{k-1}\right]=$ $[m p+2(k-2)] E\left[\left(\operatorname{tr}\left(\Psi B^{-1}\right)\right)^{k-2}\right]$.

Next, let $E\left[\left(\operatorname{tr}\left(\Psi B^{-1}\right)\right)^{k-2}\right]=E\left[\operatorname{tr}\left(\Psi B^{-1}\right) h_{3}(B)\right]$ where $h_{3}(B)=\left(\operatorname{tr}\left(\Psi B^{-1}\right)\right)^{k-3}$.

Then $h_{3}(x B)=x^{-k+3} h_{3}(B)$ so that by Corollary 2.1, we have $\quad l=-(k-3) \quad$ and $E\left[\operatorname{tr}\left(\Psi B^{-1}\right) h_{3}(B)\right]=(m p-2 l) E\left[h_{3}(B)\right]$, i.e $E\left[\left(\operatorname{tr}\left(\Psi B^{-1}\right)\right)^{k-2}\right]=$ $[m p+2(k-3)] E\left[\left(\operatorname{tr}\left(\Psi B^{-1}\right)\right)^{k-3}\right]$.

Similarly, we have
$E\left[\left(\operatorname{tr}\left(\Psi B^{-1}\right)\right)^{k-(k-2)}\right]=$

$[m p+2\{k-(k-1)\}] E\left[\left(\operatorname{tr}\left(\Psi B^{-1}\right)\right)^{k-(k-1)}\right]$

and

$E\left[\left(\operatorname{tr}\left(\Psi B^{-1}\right)\right)^{k-(k-1)}\right]=$

$[m p+2(k-k)] E\left[\left(\operatorname{tr}\left(\Psi B^{-1}\right)\right)^{k-k}\right]$

Finally, we have

$$
E\left\lfloor\left(\operatorname{tr}\left(\Psi B^{-1}\right)\right)^{k}\right\rfloor=
$$

$[m p-2(-k+1)][m p-2(-k+2)][m p-2(-k+3)]$

$\cdots[m p-2(-k+k)] E\left[\left(\operatorname{tr}\left(\Psi B^{-1}\right)\right)^{k-k}\right]$.

$=2^{k}\left[\frac{m p}{2}+(k-1)\right]\left[\frac{m p}{2}+(k-2)\right]\left[\frac{m p}{2}+(k-3)\right]$

$\cdots\left[\frac{m p}{2}+(k-k)\right]=2^{k}(m p / 2)_{\{k\}}$

2. $E\left[\operatorname{tr}\left(\Psi B^{-1}\right)^{-k}\right]=\frac{2^{-k}}{(m p / 2-1)^{\{k\}}}, m p>2 k$.

Proof. Let $E\left[\operatorname{tr}\left(\Psi B^{-1}\right)^{0}\right]=E\left[\operatorname{tr}\left(\Psi B^{-1}\right) h_{1}(B)\right]$ where $h_{1}(B)=\left[\operatorname{tr}\left(\Psi B^{-1}\right)\right]^{-1}$. Then

$h_{1}(x B)=x h_{1}(B) \quad$ so $\quad$ that $\quad l=1 \quad$ and $E\left[\operatorname{tr}\left(\Psi B^{-1}\right)^{0}\right]=(m p-2) h_{1}(B)$, i.e.,

$E\left[\operatorname{tr}\left(\Psi B^{-1}\right)^{0}\right]=(m p-2) E\left[\operatorname{tr}\left(\Psi B^{-1}\right)^{-1}\right]$

Next, let $E\left[\operatorname{tr}\left(\Psi B^{-1}\right)^{-1}\right]=E\left[\operatorname{tr}\left(\Psi B^{-1}\right) h_{2}(B)\right]$ where $h_{2}(B)=\left[\operatorname{tr}\left(\Psi B^{-1}\right)\right]^{-2}$. Then $h_{2}(x B)=x^{2} h_{2}(B) \quad$ so that $l=2$ and $E\left[\operatorname{tr}\left(\Psi B^{-1}\right)^{-1}\right]=[m p-2(2)] h_{1}(B)$, i.e., 
$E\left[\operatorname{tr}\left(\Psi B^{-1}\right)^{-1}\right]=[m p-2(2)] E\left[\operatorname{tr}\left(\Psi B^{-1}\right)^{-2}\right]$.

Next, let $E\left[\operatorname{tr}\left(\Psi B^{-1}\right)^{-2}\right]=E\left[\operatorname{tr}\left(\Psi B^{-1}\right) h_{3}(B)\right]$ where $h_{3}(B)=\left[\operatorname{tr}\left(\Psi B^{-1}\right)\right]^{-3}$. Then

$h_{3}(x B)=x^{3} h_{3}(B) \quad$ so $\quad$ that $\quad l=3 \quad$ and $E\left[\operatorname{tr}\left(\Psi B^{-1}\right)^{-2}\right]=[m p-2(3)] h_{2}(B)$, , i.e.,

$E\left[\operatorname{tr}\left(\Psi B^{-1}\right)^{-2}\right]=[m p-2(3)] E\left[\operatorname{tr}\left(\Psi B^{-1}\right)^{-3}\right]$

Similarly we have

$$
\begin{aligned}
& E\left[\operatorname{tr}\left(\Psi B^{-1}\right)^{-3}\right]=[m p-2(4)] E\left[\operatorname{tr}\left(\Psi B^{-1}\right)^{-4}\right] \\
& \cdot-\cdot-\cdot \\
& E\left[\operatorname{tr}\left(\Psi B^{-1}\right)^{-(k-2)}\right]= \\
& {[m p-2(k-1)] E\left[\operatorname{tr}\left(\Psi B^{-1}\right)^{-(k-1)}\right] \text { and }}
\end{aligned}
$$$$
E\left[\operatorname{tr}\left(\Psi B^{-1}\right)^{-(k-1)}\right]=[m p-2(k)] E\left[\operatorname{tr}\left(\Psi B^{-1}\right)^{-k}\right] .
$$

That is

$$
\begin{aligned}
& \frac{1}{(m p-2)} E\left[\operatorname{tr}\left(\Psi B^{-1}\right)^{0}\right]=E\left[\operatorname{tr}\left(\Psi B^{-1}\right)^{-1}\right], \\
& \frac{1}{[m p-2(2)]} E\left[\operatorname{tr}\left(\Psi B^{-1}\right)^{-1}\right]=E\left[\operatorname{tr}\left(\Psi B^{-1}\right)^{-2}\right],
\end{aligned}
$$$$
\frac{1}{[m p-2(3)]} E\left[\operatorname{tr}\left(\Psi B^{-1}\right)^{-2}\right]=E\left[\operatorname{tr}\left(\Psi B^{-1}\right)^{-3}\right],
$$$$
.-.-. \text {. }
$$$$
\frac{1}{[m p-2(k-1)]} E\left[\operatorname{tr}\left(\Psi B^{-1}\right)^{-(k-2)}\right]=
$$$$
E\left[\operatorname{tr}\left(\Psi B^{-1}\right)^{-(k-1)}\right]
$$

and

$$
\frac{1}{[m p-2(k)]} E\left[\operatorname{tr}\left(\Psi B^{-1}\right)^{-(k-1)}\right]=E\left[\operatorname{tr}\left(\Psi B^{-1}\right)^{-k}\right] .
$$

In general for any $k>0$, we have

$$
\begin{aligned}
& E\left\lfloor\operatorname{tr}\left(\Psi B^{-1}\right)^{-k}\right\rfloor= \\
& \frac{1}{m p-2(k)} \times \frac{1}{m p-2(2)} \times \frac{1}{m p-2} E\left[\operatorname{tr}\left(\Psi B^{-1}\right)^{0}\right] \\
& =\frac{2^{-k}}{(m p / 2-1)^{\{k\}}}, m p>2 k \text {. }
\end{aligned}
$$

Proof.

Let

$E\left\lfloor\left(\operatorname{tr}\left(\Psi B^{-1}\right)\right)^{k} \operatorname{tr} B\right\rfloor=E\left[\operatorname{tr}\left(\Psi B^{-1}\right) h_{1}(B)\right] \quad$ where $h_{1}(B)=\left(\operatorname{tr}\left(\Psi B^{-1}\right)\right)^{k-1} \operatorname{tr} B$.

Then

$h_{1}(x B)=x^{-k+2} h_{1}(B)$ so that by Corollary 2.1 , we have $\quad l=-k+2 \quad$ and $E\left[\operatorname{tr}\left(\Psi B^{-1}\right) h_{1}(B)\right]=[m p-2(-k+2)] E\left[h_{1}(B)\right]$, i.e.

$E\left[\left(\operatorname{tr}\left(\Psi B^{-1}\right)\right)^{k} \operatorname{tr} B\right\rfloor=$

$[m p+2(k-2)] E\left[\left(\operatorname{tr}\left(\Psi B^{-1}\right)\right)^{k-1} \operatorname{tr} B\right]$.

Similarly,

$$
\begin{aligned}
& E\left[\left(\operatorname{tr}\left(\Psi B^{-1}\right)\right)^{k-1} \operatorname{tr} B\right]= \\
& {[m p+2(k-3)] E\left[\left(\operatorname{tr}\left(\Psi B^{-1}\right)\right)^{k-2} \operatorname{tr} B\right]} \\
& E\left[\left(\operatorname{tr}\left(\Psi B^{-1}\right)\right)^{k-2} \operatorname{tr} B\right]= \\
& {[m p+2(k-4)] E\left[\left(t r\left(\Psi B^{-1}\right)\right)^{k-3} \operatorname{tr} B\right]} \\
& E\left[\left(t r\left(\Psi B^{-1}\right)\right)^{k-3} \operatorname{tr} B\right]= \\
& {[m p+2(k-5)] E\left[\left(\operatorname{tr}\left(\Psi B^{-1}\right)\right)^{k-4} \operatorname{tr} B\right]}
\end{aligned}
$$




$$
E\left[\left(\operatorname{tr}\left(\Psi B^{-1}\right)\right)^{k-(k-3)} \operatorname{tr} B\right]=
$$

$[m p+2(k-(k-1))] E\left[\left(\operatorname{tr}\left(\Psi B^{-1}\right)\right)^{k-(k-2)} \operatorname{tr} B\right]$

$$
E\left[\left(\operatorname{tr}\left(\Psi B^{-1}\right)\right)^{k-(k-2)} \operatorname{tr} B\right]=
$$

$[m p+2(k-k)] E\left[\left(\operatorname{tr}\left(\Psi B^{-1}\right)\right)^{k-(k-1)} \operatorname{tr} B\right]^{\text {and }}$

Proceeding thus,

$$
\begin{aligned}
& E\left[\left(\operatorname{tr}\left(\Psi B^{-1}\right)\right)^{k} \operatorname{tr} B\right\rfloor \\
&= {[m p+2(k-2)][m p+2(k-3)] \cdots } \\
& \times \cdots[m p+2(k-(k-1))][m p+2(k-k)] E \\
& \\
& {\left[\left(\operatorname{tr}\left(\Psi B^{-1}\right)\right)^{k-(k-1)} \operatorname{tr} B\right], }
\end{aligned}
$$

which can be written as

$$
\begin{aligned}
& E\left[\left(\operatorname{tr}\left(\Psi B^{-1}\right)\right)^{k} \operatorname{tr} B\right] \\
= & 2^{k-1} \times\left[\frac{m p}{2}+(k-2)\right]\left[\frac{m p}{2}+(k-3)\right] \cdots \\
\times & \cdots\left[\frac{m p}{2}+2\{k-(k-1)\}\right]\left[\frac{m p}{2}+2(k-k)\right] \\
& E\left[\left(\operatorname{tr}\left(\Psi B^{-1}\right)\right)^{k-(k-1)} \operatorname{tr} B\right]
\end{aligned}
$$

which can be written as

$$
\begin{aligned}
& E\left\lfloor\left(\operatorname{tr}\left(\Psi B^{-1}\right)\right)^{k} \operatorname{tr} B\right\rfloor= \\
& 2^{k-1}\left(\frac{m p}{2}\right)_{\{k-1\}} E\left[\left(\operatorname{tr}\left(\Psi B^{-1}\right)\right)^{k-(k-1)} \operatorname{tr} B\right] . \\
& \text { 4. } E\left[\left(t r \Psi^{-1} B\right)^{-k} \operatorname{tr} B\right\rfloor= \\
& \frac{1}{2^{k}(m-p-1)(m p / 2)_{\{k\}}} \operatorname{tr} \Psi, m>p+1 .
\end{aligned}
$$

Proof.Let $E\left[\operatorname{tr}\left(\Psi^{-1} B\right)^{0} \operatorname{tr} B\right]=E\left[\left(\operatorname{tr} \Psi^{-1} B\right) h_{1}(B)\right]$ where $h_{1}(B)=\left(\operatorname{tr} \Psi^{-1} B\right)^{-1} \operatorname{tr} B$. Then $h_{1}(x B)=x^{0} h_{1}(B) \quad$ so $\quad$ that $\quad l=0 \quad$ and $E\left[\left(\operatorname{tr} \Psi^{-1} B\right) h_{1}(B)\right]=(m p-2 \times 0) E\left[h_{1}(B)\right]$, i.e.

$E\left[\operatorname{tr}\left(\Psi^{-1} B\right)^{0} \operatorname{tr} B\right]=m p E\left[\left(\operatorname{tr} \Psi^{-1} B\right)^{-1} \operatorname{tr} B\right]$. Next let $E\left[\operatorname{tr}\left(\Psi^{-1} B\right)^{-1} \operatorname{tr} B\right]=E\left[\left(\operatorname{tr} \Psi^{-1} B\right)^{1} h_{2}(B)\right] \quad$ where $h_{2}(B)=\left(\operatorname{tr} \Psi^{-1} B\right)^{-2} \operatorname{tr} B$. Then

$h_{2}(x B)=\left(\operatorname{tr} \Psi^{-1} x B\right)^{-2} \operatorname{tr}(x B)=x^{-1} h_{2}(B) \quad$ so that $l=-1$ and

$E\left[\left(\operatorname{tr} \Psi^{-1} B\right) h_{2}(B)\right]=[m p-2(-1)] E\left[h_{2}(B)\right]$, i.e.

$E\left[\left(\operatorname{tr} \Psi^{-1} B\right)^{-1} \operatorname{tr} B\right]=$

$[m p-2(-1)] E\left[\left(\operatorname{tr} \Psi^{-1} B\right)^{-2} \operatorname{tr} B\right]^{\cdot}$

Next let $E\left[\left(\operatorname{tr} \Psi^{-1} B\right)^{-2} \operatorname{tr} B\right]=E\left[\left(\operatorname{tr} \Psi^{-1} B\right) h_{3}(B)\right]$ where $h_{3}(B)=\left(\operatorname{tr} \Psi^{-1} B\right)^{-3} \operatorname{tr} B$. Then

$h_{3}(x B)=\left(\operatorname{tr} \Psi^{-1} x B\right)^{-3} \operatorname{tr}(x B)=x^{-2} h_{3}(B) \quad$ so that $l=-2$ and

$E\left[\left(\operatorname{tr} \Psi^{-1} B\right) h_{3}(B)\right]=[m p-2(-2)] E\left[h_{3}(B)\right]$, i.e.

$E\left[\left(\operatorname{tr} \Psi^{-1} B\right)^{-2} \operatorname{tr} B\right]=$

$[m p-2(-2)] E\left[\left(\operatorname{tr} \Psi^{-1} B\right)^{-3} \operatorname{tr} B\right]^{\cdot}$

Similarly,

$$
\begin{aligned}
& E\left[\left(\operatorname{tr} \Psi^{-1} B\right)^{-(k-2)} \operatorname{tr} B\right]= \\
& {[m p-2\{-(k-2)\}] E\left[\left(\operatorname{tr} \Psi^{-1} B\right)^{-(k-1)} \operatorname{tr} B\right]} \\
& E\left[\left(\operatorname{tr} \Psi^{-1} B\right)^{-(k-1)} \operatorname{tr} B\right]= \\
& {[m p-2\{-(k-1)\}] E\left[\operatorname{tr}\left(\Psi^{-1} B\right)^{-k} \operatorname{tr} B\right]}
\end{aligned}
$$

Proceeding thus, we have 
$m p E\left[\operatorname{tr}\left(\Psi^{-1} B\right)^{0} \operatorname{tr} B\right]=E\left[\left(\operatorname{tr} \Psi^{-1} B\right)^{-1} \operatorname{tr} B\right]$,

$\frac{1}{[m p-2(-1)]} E\left[\left(\operatorname{tr} \Psi^{-1} B\right)^{-1} \operatorname{tr} B\right]=$

$E\left[\left(\operatorname{tr} \Psi^{-1} B\right)^{-2} \operatorname{tr} B\right]$,

$\frac{1}{[m p-2\{-(k-2)\}]} E\left[\left(\operatorname{tr} \Psi^{-1} B\right)^{-(k-2)} \operatorname{tr} B\right]=$

$E\left[\left(\operatorname{tr} \Psi^{-1} B\right)^{-(k-1)} \operatorname{tr} B\right]$

$\frac{1}{[m p-2\{-(k-1)\}]} E\left[\left(\operatorname{tr} \Psi^{-1} B\right)^{-(k-1)} \operatorname{tr} B\right]=$

$E\left[\operatorname{tr}\left(\Psi^{-1} B\right)^{-k} \operatorname{tr} B\right]$.

Finally, we have

$$
\begin{aligned}
& E\left[\left(\operatorname{tr} \Psi^{-1} B\right)^{-k} \operatorname{tr} B\right] \\
& =\frac{1}{2^{k}\left[\frac{m p}{2}+(k-1)\right]} \times \frac{1}{\left[\frac{m p}{2}+(k-2)\right]} \times \cdots \times
\end{aligned}
$$

$\frac{1}{\left[\frac{m p}{2}+2\right]} \times \frac{1}{\left[\frac{m p}{2}+1\right]} \times \frac{1}{\left[\frac{m p}{2}\right]} E\left[\operatorname{tr}\left(\Psi^{-1} B\right)^{0} \operatorname{tr} B\right]$,

which can be written as

$$
E\left\lfloor\left(\operatorname{tr} \Psi^{-1} B\right)^{-k} \operatorname{tr} B\right\rfloor=
$$

$\frac{1}{2^{k}(m p / 2)_{\{k\}}} E(t r B), m>p+1$.

5. $E\left\lfloor\left(\operatorname{tr}\left(\Psi^{-1} B\right)\right)^{k} \operatorname{tr}\left(B^{-1}\right)\right\rfloor=$

$2^{k} m((m p / 2)+1)_{\{k\}} t r \Psi^{-1}$.

Proof. Let

$$
\begin{aligned}
& E\left[\left(\operatorname{tr}\left(\Psi B^{-1}\right)\right)^{k} \operatorname{tr}\left(B^{-1}\right)\right] \\
= & E\left[\operatorname{tr}\left(\Psi B^{-1}\right) h_{1}(B)\right]
\end{aligned}
$$

where $h_{1}(B)=\left(\operatorname{tr}\left(\Psi B^{-1}\right)\right)^{k-1} \operatorname{tr}\left(B^{-1}\right)$.
Then $h_{1}(x B)=x^{-k} h_{1}(B)$

so that by Corollary 2.1 , we have $l=-k$ and $E\left[\operatorname{tr}\left(\Psi B^{-1}\right) h_{1}(B)\right]=$ $[m p-2(-k+2)] E\left[h_{1}(B)\right]$,

$$
E\left[\left(\operatorname{tr}\left(\Psi B^{-1}\right)\right)^{k} \operatorname{tr}\left(B^{-1}\right)\right]=
$$

$[m p+2 k] E\left[\left(\operatorname{tr}\left(\Psi B^{-1}\right)\right)^{k-1} \operatorname{tr}\left(B^{-1}\right)\right]$.

Similarly,

$$
\begin{aligned}
& E\left[\left(\operatorname{tr}\left(\Psi B^{-1}\right)\right)^{k-1} \operatorname{tr}\left(B^{-1}\right)\right]= \\
& {[m p+2(k-1)] E\left[\left(\operatorname{tr}\left(\Psi B^{-1}\right)\right)^{k-2} \operatorname{tr}\left(B^{-1}\right)\right]}
\end{aligned}
$$

and

$$
\begin{aligned}
& E\left[\left(\operatorname{tr}\left(\Psi B^{-1}\right)\right)^{k-(k-1)} \operatorname{tr}\left(B^{-1}\right)\right]= \\
& {[m p+2\{k-(k-1)\}] E\left[\left(\operatorname{tr}\left(\Psi B^{-1}\right)\right)^{k-k} \operatorname{tr}\left(B^{-1}\right)\right]}
\end{aligned} .
$$

Proceeding thus,

$$
\begin{aligned}
& E\left[\left(\operatorname{tr}\left(\Psi B^{-1}\right)\right)^{k} \operatorname{tr}\left(B^{-1}\right)\right\rfloor \\
= & {[m p+2 k][m p+2(k-1)] \cdots } \\
\ldots & {[m p+2\{k-(k+1)\}] E\left[\left(\operatorname{tr}\left(\Psi B^{-1}\right)\right)^{k-k} \operatorname{tr}\left(B^{-1}\right)\right], }
\end{aligned}
$$

which simplifies to

$$
E\left[\left(\operatorname{tr}\left(\Psi^{-1} B\right)\right)^{k} \operatorname{tr}\left(B^{-1}\right)\right\rfloor=2^{k} m\left(\frac{m p}{2}+1\right)_{\{k\}} E t r B^{-1} .
$$

Since, $\operatorname{Etr} B^{-1}=\operatorname{tr} E\left(B^{-1}\right)=\operatorname{tr} E(A)=m \operatorname{tr} \Sigma=m \operatorname{tr} \Psi^{-1}$, we have

$$
\begin{aligned}
& E\left[\left(\operatorname{tr}\left(\Psi^{-1} B\right)\right)^{k} \operatorname{tr}\left(B^{-1}\right)\right\rfloor= \\
& 2^{k} m((m p / 2)+1)_{\{k\}} \operatorname{tr} \Psi^{-1} . \\
& \text { 6. } E\left[\left(\operatorname{tr} \Psi^{-1} B\right)^{k} \operatorname{tr}\left(B^{-1}\right)\right]=\frac{m t r \Psi^{-1}}{2^{k}\left(\frac{m p}{2}+2\right)} .
\end{aligned}
$$


Proof. Let

$E\left[\left(\operatorname{tr} \Psi^{-1} B\right)^{0} \operatorname{tr}\left(B^{-1}\right)\right]=E\left[\left(\operatorname{tr} \Psi^{-1} B\right) h_{1}(B)\right]$

where $h_{1}(B)=\left(\operatorname{tr} \Psi^{-1} B\right)^{-1} \operatorname{tr}\left(B^{-1}\right)$.

Then $h_{1}(x B)=\left(\operatorname{tr} \Psi^{-1} x B\right)^{-1} \operatorname{tr}\left((x B)^{-1}\right)=x^{-2} h_{1}(B)$

so that $\quad l=-2 \quad$ and

$E\left[\left(\operatorname{tr} \Psi^{-1} B\right) h_{1}(B)\right]=[m p-2 \times(-2)] E\left[h_{1}(B)\right]$, i.e.,

$E\left[\operatorname{tr}\left(B^{-1}\right)\right]=[m p+2(2)] E\left[\left(\operatorname{tr} \Psi^{-1} B\right)^{-1} \operatorname{tr}\left(B^{-1}\right)\right]$.

Let $\quad E\left[\left(\operatorname{tr} \Psi^{-1} B\right)^{-1} \operatorname{tr}\left(B^{-1}\right)\right]=E\left[\left(\operatorname{tr} \Psi^{-1} B\right) h_{2}(B)\right]$ where $\quad h_{2}(B)=\left(\operatorname{tr} \Psi^{-1} B\right)^{-2} \operatorname{tr}\left(B^{-1}\right) . \quad$ Then $h_{2}(x B)=\left(\operatorname{tr} \Psi^{-1} x B\right)^{-2} \operatorname{tr}\left((x B)^{-1}\right)=x^{-3} h_{2}(B)$ so that $l=-3$ and

$E\left[\left(\operatorname{tr} \Psi^{-1} B\right) h_{2}(B)\right]=[m p-2 \times(-3)] E\left[h_{2}(B)\right]$, i.e.,

$E\left[\left(\operatorname{tr} \Psi^{-1} B\right)^{-1} \operatorname{tr}\left(B^{-1}\right)\right]=$

$[m p+2(3)] E\left[\left(\operatorname{tr} \Psi^{-1} B\right)^{-2} \operatorname{tr}\left(B^{-1}\right)\right]^{\cdot}$

Similarly

$E\left[\left(\operatorname{tr} \Psi^{-1} B\right)^{-(k-2)} \operatorname{tr}\left(B^{-1}\right)\right]=$

$[m p+2(k)] E\left[\left(\operatorname{tr} \Psi^{-1} B\right)^{-(k-1)} \operatorname{tr}\left(B^{-1}\right)\right]^{\prime}$

$E\left[\left(\operatorname{tr} \Psi^{-1} B\right)^{-(k-1)} \operatorname{tr}\left(B^{-1}\right)\right]=$

$[m p+2(k+1)] E\left[\left(\operatorname{tr} \Psi^{-1} B\right)^{-k} \operatorname{tr}\left(B^{-1}\right)\right]$

That is

$$
\begin{aligned}
& E\left[\left(\operatorname{tr} \Psi^{-1} B\right)^{-k} \operatorname{tr}\left(B^{-1}\right)\right]= \\
& \frac{1}{[m p+2(k+1)]} E\left[\left(\operatorname{tr} \Psi^{-1} B\right)^{-(k-1)} \operatorname{tr}\left(B^{-1}\right)\right],
\end{aligned}
$$

$$
\begin{aligned}
& E\left[\left(\operatorname{tr} \Psi^{-1} B\right)^{-(k-1)} \operatorname{tr}\left(B^{-1}\right)\right]= \\
& \frac{1}{[m p+2(k)]} E\left[\left(\operatorname{tr} \Psi^{-1} B\right)^{-(k-2)} \operatorname{tr}\left(B^{-1}\right)\right]
\end{aligned}
$$$$
.-.-.- \text {. }
$$$$
E\left[\left(\operatorname{tr} \Psi^{-1} B\right)^{-2} \operatorname{tr}\left(B^{-1}\right)\right]=
$$$$
\frac{1}{[m p+2(3)]} E\left[\left(\operatorname{tr} \Psi^{-1} B\right)^{-1} \operatorname{tr}\left(B^{-1}\right)\right]
$$$$
E\left[\left(\operatorname{tr} \Psi^{-1} B\right)^{-1} \operatorname{tr}\left(B^{-1}\right)\right]=\frac{1}{[m p+2(2)]} E\left[\operatorname{tr}\left(B^{-1}\right)\right]
$$

Finally, we have

$$
\begin{aligned}
& E\left[\left(\operatorname{tr} \Psi^{-1} B\right)^{-k} \operatorname{tr}\left(B^{-1}\right)\right] \\
& =\frac{1}{[m p+2(k+1)][m p+2(k)]} \cdots \\
& \ldots \frac{1}{[m p+2(3)]} \frac{1}{[m p+2(2)]} E\left[\operatorname{tr}\left(B^{-1}\right)\right] \\
& =\frac{1}{2^{k} \times \frac{m p}{2}+(k+1)} \times \frac{1}{\frac{m p}{2}+k} \times \cdots \\
& \ldots \times \frac{1}{\frac{m p}{2}+3} \times \frac{1}{\frac{m p}{2}+2} \operatorname{tr}\left[E\left(B^{-1}\right)\right]
\end{aligned}
$$

which simplifies to

$$
\begin{aligned}
& E\left[\left(t r \Psi^{-1} B\right)^{-k} \operatorname{tr}\left(B^{-1}\right)\right]= \\
& \frac{1}{2^{k}} \times \frac{1}{\left(\frac{m p}{2}+2\right)} \operatorname{tr}[E(A)],
\end{aligned}
$$

which can be evaluated to

$$
E\left[\left(\operatorname{tr} \Psi^{-1} B\right)^{k} \operatorname{tr}\left(B^{-1}\right)\right]=\frac{m t r \Sigma}{2^{k}\left(\frac{m p}{2}+2\right)} .
$$

7. $E\left[\left(\operatorname{tr}\left(\Psi B^{-1}\right)\right)^{k} \operatorname{tr}\left(\Psi^{-1} B\right)\right]=$ $\frac{2^{k-2} p}{m-p-1}((m p / 2)+1)_{\{k-2\}}, m>p+1$. 


\section{Proof.Let}

$$
E\left[\left(\operatorname{tr}\left(\Psi B^{-1}\right)\right)^{k} \operatorname{tr}\left(\Psi^{-1} B\right)\right]=E\left[\operatorname{tr}\left(\Psi B^{-1}\right) h_{1}(B)\right]
$$

where $\quad h_{1}(B)=\left(\operatorname{tr}\left(\Psi B^{-1}\right)\right)^{k-1} \operatorname{tr}\left(\Psi^{-1} B\right)$. Then $h_{1}(x B)=x^{-k+2} h_{1}(B)$ so that by Corollary 2.1, we have $l=-k+2 \quad$ and $E\left[\operatorname{tr}\left(\Psi B^{-1}\right) h_{1}(B)\right]=[m p-2(-k+2)] E\left[h_{1}(B)\right]$, i.e.

$$
\begin{aligned}
& E\left[\left(\operatorname{tr}\left(\Psi B^{-1}\right)\right)^{k} \operatorname{tr}\left(\Psi^{-1} B\right)\right]= \\
& {[m p+2(k-2)] E\left[\left(\operatorname{tr}\left(\Psi B^{-1}\right)\right)^{k-1} \operatorname{tr}\left(\Psi^{-1} B\right)\right] .}
\end{aligned}
$$

Similarly, we have

$$
\begin{aligned}
& E\left[\left(\operatorname{tr}\left(\Psi B^{-1}\right)\right)^{k-1} \operatorname{tr}\left(\Psi^{-1} B\right)\right]= \\
& {[m p+2(k-3)] E\left[\left(\operatorname{tr}\left(\Psi B^{-1}\right)\right)^{k-2} \operatorname{tr}\left(\Psi^{-1} B\right)\right]} \\
& E\left[\left(\operatorname{tr}\left(\Psi B^{-1}\right)\right)^{k-(k-2)} \operatorname{tr}\left(\Psi^{-1} B\right)\right]= \\
& {[m p+2\{k-(k)\}] E\left[\left(\operatorname{tr}\left(\Psi B^{-1}\right)\right)^{k-(k-1)} \operatorname{tr}\left(\Psi^{-1} B\right)\right]}
\end{aligned}
$$

and

$$
\begin{aligned}
& E\left[\left(\operatorname{tr}\left(\Psi B^{-1}\right)\right)^{k-(k-1)} \operatorname{tr}\left(\Psi^{-1} B\right)\right]= \\
& {[m p+2\{k-(k+1)\}] E\left[\left(\operatorname{tr}\left(\Psi B^{-1}\right)\right)^{k-k} \operatorname{tr}\left(\Psi^{-1} B\right)\right] .}
\end{aligned}
$$

Finally we have

$$
\begin{aligned}
& E\left[\left(\operatorname{tr}\left(\Psi B^{-1}\right)\right)^{k} \operatorname{tr}\left(\Psi^{-1} B\right)\right\rfloor \\
& =[m p+2(k-2)][m p+2(k-3)] \cdots \\
& \ldots[m p+2\{k-(k+1)\}] E\left[\left(\operatorname{tr}\left(\Psi B^{-1}\right)\right)^{k-k} \operatorname{tr}\left(\Psi^{-1} B\right)\right] .
\end{aligned}
$$

which can be simplified to be

$$
\begin{aligned}
& E\left[\left(\operatorname{tr}\left(\Psi B^{-1}\right)\right)^{k} \operatorname{tr}\left(\Psi^{-1} B\right)\right\rfloor= \\
& 2^{k-2} \frac{((m p / 2)+1)_{\{k-2\}} t r \Psi^{-1} E(B)}{m-p-1}, m>p+1,
\end{aligned}
$$

Since $\operatorname{tr} \Psi^{-1} E(B)=\operatorname{tr} \Psi^{-1}\left(\frac{1}{m-p-1} \Psi\right)=$

$\frac{1}{m-p-1} \operatorname{trI} p=\frac{p}{m-p-1}$,

we have

$$
\begin{aligned}
& E\left[\left(\operatorname{tr}\left(\Psi B^{-1}\right)\right)^{k} \operatorname{tr}\left(\Psi^{-1} B\right)\right\rfloor= \\
& \frac{2^{k-2} p}{m-p-1}((m p / 2)+1)_{\{k-2\}}, m>p+1 .
\end{aligned}
$$

8. $E\left[\left(\operatorname{tr}\left(\Psi B^{-1}\right)\right)^{-k} \operatorname{tr}\left(\Psi^{-1} B\right)\right]=$

$$
\frac{2^{-k} p}{(m-p-1)((m p / 2)-k-1)_{\{k\}}},
$$

$m>\max \{p+1,4 / p\}$

Proof. Let

$$
E\left[\left(\operatorname{tr}\left(\Psi B^{-1}\right)\right)^{0} \operatorname{tr}\left(\Psi^{-1} B\right)\right]=E\left[\left(\operatorname{tr}\left(\Psi B^{-1}\right)\right) h_{1}(B)\right]
$$

where $\quad h_{1}(B)=\left(\operatorname{tr}\left(\Psi B^{-1}\right)\right)^{-1} \operatorname{tr}\left(\Psi^{-1} B\right) . \quad$ Then $h_{1}(x B)=x^{2} h_{1}(B) \quad$ so that $\quad l=2 \quad$ and $E\left[\left(\operatorname{tr}\left(\Psi B^{-1}\right)\right)^{0} h_{1}(B)\right]=[m p-2(2)] E\left[h_{1}(B)\right]$, i.e.,

$E\left[\left(\operatorname{tr}\left(\Psi B^{-1}\right)\right)^{0} \operatorname{tr}\left(\Psi B^{-1}\right)\right]=$

$[m p-2(2)] E\left[\left(\operatorname{tr}\left(\Psi B^{-1}\right)\right)^{-1} \operatorname{tr}\left(\Psi^{-1} B\right)\right]$

Next let $E\left[\left(\operatorname{tr}\left(\Psi B^{-1}\right)\right)^{-1} \operatorname{tr}\left(\Psi^{-1} B\right)\right]=E\left[\left(\operatorname{tr}\left(\Psi B^{-1}\right)\right) h_{2}(B)\right]$ where $\quad h_{2}(B)=\left(\operatorname{tr}\left(\Psi B^{-1}\right)\right)^{-2} \operatorname{tr}\left(\Psi^{-1} B\right)$. Then $h_{2}(x B)=x^{3} h_{2}(B) \quad$ so that $\quad l=3 \quad$ and $E\left[\left(\operatorname{tr}\left(\Psi B^{-1}\right)\right) h_{2}(B)\right\rfloor=[m p-2(3)] E\left[h_{2}(B)\right]$, i.e.,

$E\left[\left(\operatorname{tr}\left(\Psi B^{-1}\right)\right)^{-1} \operatorname{tr}\left(\Psi^{-1} B\right)\right]=$

$[m p-2(3)] E\left[\left(\operatorname{tr}\left(\Psi B^{-1}\right)\right)^{-2} \operatorname{tr}\left(\Psi^{-1} B\right)\right]$

Similarly 


$$
\begin{aligned}
& E\left[\left(\operatorname{tr}\left(\Psi B^{-1}\right)\right)^{-(k-2)} \operatorname{tr}\left(\Psi^{-1} B\right)\right]= \\
& {[m p-2(k)] E\left[\left(\operatorname{tr}\left(\Psi B^{-1}\right)\right)^{-(k-1)} \operatorname{tr}\left(\Psi^{-1} B\right)\right]}
\end{aligned}
$$

and

$E\left[\left(\operatorname{tr}\left(\Psi B^{-1}\right)\right)^{-(k-1)} \operatorname{tr}\left(\Psi^{-1} B\right)\right]=$

$[m p-2(k+1)] E\left[\left(\operatorname{tr}\left(\Psi B^{-1}\right)\right)^{-k} \operatorname{tr}\left(\Psi^{-1} B\right)\right]$

That is

$$
\begin{aligned}
& E\left[\left(\operatorname{tr}\left(\Psi B^{-1}\right)\right)^{-k} \operatorname{tr}\left(\Psi^{-1} B\right)\right]= \\
& \frac{1}{[m p-2(k+1)]} E\left[\left(\operatorname{tr}\left(\Psi B^{-1}\right)\right)^{-(k-1)} \operatorname{tr}\left(\Psi^{-1} B\right)\right] \\
& E\left[\left(\operatorname{tr}\left(\Psi B^{-1}\right)\right)^{-(k-1)} \operatorname{tr}\left(\Psi^{-1} B\right)\right]= \\
& \frac{1}{[m p-2(k)]} E\left[\left(\operatorname{tr}\left(\Psi B^{-1}\right)\right)^{-(k-2)} \operatorname{tr}\left(\Psi^{-1} B\right)\right], \\
& .-.-.-. \\
& E\left[\left(\operatorname{tr}\left(\Psi B^{-1}\right)\right)^{-2} \operatorname{tr}\left(\Psi^{-1} B\right)\right]= \\
& \frac{1}{[m p-2(3)]} E\left[\left(\operatorname{tr}\left(\Psi B^{-1}\right)\right)^{-1} \operatorname{tr}\left(\Psi^{-1} B\right)\right] \\
& E\left[\left(\operatorname{tr}\left(\Psi B^{-1}\right)\right)^{-1} \operatorname{tr}\left(\Psi^{-1} B\right)\right]= \\
& \frac{1}{[m p-2(2)]} E\left[\operatorname{tr}\left(\Psi B^{-1}\right)\right] .
\end{aligned}
$$

Finally we have

$$
\begin{aligned}
& E\left[\left(\operatorname{tr}\left(\Psi B^{-1}\right)\right)^{-k} \operatorname{tr}\left(\Psi^{-1} B\right)\right\rfloor \\
& =\frac{1}{[m p-2(k+1)]} \times \frac{1}{[m p-2(k)]} \times \cdots \\
& \times \frac{1}{[m p-2(3)]} \times \frac{1}{[m p-2(2)]} E\left[\operatorname{tr}\left(\Psi^{-1} B\right)\right],
\end{aligned}
$$

which can be written as

$$
\begin{aligned}
& E\left[\left(\operatorname{tr}\left(\Psi B^{-1}\right)\right)^{-k} \operatorname{tr}\left(\Psi^{-1} B\right)\right\rfloor \\
= & \frac{1}{2^{k}[(m p / 2)-(k+1)]} \times \frac{1}{[(m p / 2)-k]} \times \cdots \\
\times & \frac{1}{[(m p / 2)-3]} \times \frac{1}{[(m p / 2)-2]} E\left[\operatorname{tr}\left(\Psi^{-1} B\right)\right]
\end{aligned}
$$

which can be represented by

$$
\begin{aligned}
& E\left[\left(\operatorname{tr}\left(\Psi B^{-1}\right)\right)^{-k} \operatorname{tr}\left(\Psi^{-1} B\right)\right\rfloor= \\
& \frac{1}{2^{k}} \frac{1}{((m p / 2)-2)^{\{k\}}} E\left[\operatorname{tr}\left(\Psi^{-1} B\right)\right],
\end{aligned}
$$

Since,

$$
\begin{aligned}
& E\left[\operatorname{tr}\left(\Psi^{-1} B\right)\right]=\operatorname{tr} \Psi^{-1} E(B)=\operatorname{tr} \Psi^{-1}\left(\frac{1}{m-p-1} \Psi\right) \\
& =\frac{\operatorname{tr} I_{p}}{m-p-1}=\frac{p}{m-p-1}, \\
& E\left[\left(\operatorname{tr}\left(\Psi B^{-1}\right)\right)^{-k} \operatorname{tr}\left(\Psi^{-1} B\right)\right]= \\
& \frac{1}{2^{k}} \frac{1}{((m p / 2)-2)^{\{k\}}} \frac{p}{m-p-1} .
\end{aligned}
$$

Since,

$$
\begin{aligned}
& x^{\{n\}}=x(x-1) \cdots(x-n+1)= \\
& (x-n+1)(x-n+2) \cdots(x-1) x=(x-n+1)_{\{n\}},
\end{aligned}
$$

we have $((m p / 2)-2)^{\{k\}}=((m p / 2)-2-k+1)_{\{k\}}$, and the above can be represented as

$$
E\left[\left(\operatorname{tr}\left(\Psi B^{-1}\right)\right)^{-k} \operatorname{tr}\left(\Psi^{-1} B\right)\right\rfloor=
$$

$\frac{2^{-k} p}{(m-p-1)((m p / 2)-k-1)_{\{k\}}}$,

$m>\max \{p+1,4 / p\}$.

9. $E\left\lfloor\left(\operatorname{tr}\left(\Psi B^{-1}\right)\right)^{k}\left|B^{-1}\right|^{h}\right\rfloor=$

$2^{k+h p}((m p / 2)+h p)_{\{k\}} \frac{\Gamma_{p}(m / 2+h)}{\Gamma_{p}(m / 2)}|\Psi|^{-h}$.

Proof.

Let

$E\left[\left(\operatorname{tr}\left(\Psi B^{-1}\right)\right)^{k}\left|B^{-1}\right|^{h}\right]=E\left[\left(\operatorname{tr}\left(\Psi B^{-1}\right)\right)^{k-1} h_{1}(B)\right]$ 
where $\quad h_{1}(B)=\left(\operatorname{tr}\left(\Psi B^{-1}\right)\right)^{k-1}\left|B^{-1}\right|^{h} . \quad$ Then $h_{1}(x B)=x^{-k-h p+1} h_{1}(B)$ so that by Corollary 2.1, we have $\quad l=-(k+h p-1) \quad$ and $E\left[\operatorname{tr}\left(\Psi B^{-1}\right) h_{1}(B)\right\rfloor=$ $[m p-2\{-(k+h p-1)\}] E\left[h_{1}(B)\right]$, $E\left[\left(\operatorname{tr}\left(\Psi B^{-1}\right)\right)^{k}\left|B^{-1}\right|^{h}\right]=$ $[m p+2(k-1+h p)] E\left[\left(\operatorname{tr}\left(\Psi B^{-1}\right)\right)^{k-1}\left|B^{-1}\right|^{h}\right]$.

Similarly we have $E\left[\left(\operatorname{tr}\left(\Psi B^{-1}\right)\right)^{k-1}\left|B^{-1}\right|^{h}\right]=$
$[m p+2(k-2+h p)] E\left[\left(\operatorname{tr}\left(\Psi B^{-1}\right)\right)^{k-2}\left|B^{-1}\right|^{h}\right]$, $E\left[\left(\operatorname{tr}\left(\Psi B^{-1}\right)\right)^{k-(k-2)}\left|B^{-1}\right|^{h}\right]=$ $[m p+2\{k-(k-1)+h p\}] E\left[\left(\operatorname{tr}\left(\Psi B^{-1}\right)\right)^{k-(k-1)}\left|B^{-1}\right|^{h}\right]$ and

$$
\begin{aligned}
& E\left[\left(\operatorname{tr}\left(\Psi B^{-1}\right)\right)^{k-(k-1)}\left|B^{-1}\right|^{h}\right]= \\
& {[m p+2(k-k+h p)] E\left[\left(\operatorname{tr}\left(\Psi B^{-1}\right)\right)^{k-k}\left|B^{-1}\right|^{h}\right]}
\end{aligned}
$$

Proceeding thus, we have

$$
E\left\lfloor\left(\operatorname{tr}\left(\Psi B^{-1}\right)\right)^{k}\left|B^{-1}\right|^{h}\right\rfloor
$$

$=[m p+2(k-1+h p)][m p+2(k-2+h p)] \cdots$

$[m p+2\{k-(k-1)+h p\}][m p+2(k-k+h p)]$.

$E\left[\left(\operatorname{tr}\left(\Psi B^{-1}\right)\right)^{k-k}\left|B^{-1}\right|^{h}\right]$

$$
E\left\lfloor\left(\operatorname{tr}\left(\Psi B^{-1}\right)\right)^{k}\left|B^{-1}\right|^{h}\right\rfloor
$$

$=[(m p / 2)+(k-1+h p)][(m p / 2)+(k-2+h p)] \cdots$

$[(m p / 2)+\{k-(k-1)+h p\}][(m p / 2)+(k-k+h p)]$ $E\left[\left(\operatorname{tr}\left(\Psi B^{-1}\right)\right)^{k-k}\left|B^{-1}\right|^{h}\right]$ which can be represented by

$$
\begin{aligned}
& E\left[\left(\operatorname{tr}\left(\Psi B^{-1}\right)\right)^{k}\left|B^{-1}\right|^{h}\right]= \\
& 2^{k}((m p / 2)+h p)_{\{k\}} E\left[|B|^{-h}\right]
\end{aligned}
$$

Now, from ( ) we have

$$
E\left(|B|^{-h}\right)=\frac{\Gamma_{p}(m / 2+h)}{2^{-h p} \Gamma_{p}(m / 2)}|\Psi|^{-h} .
$$

Since $B^{-1}=A$, we also have

$$
E\left(|B|^{-h}\right)=E\left(|A|^{h}\right)=2^{h p} \frac{\Gamma_{p}((m / 2)+h)}{\Gamma_{p}(m / 2)}|\Sigma|^{h},
$$

which is equivalent to what we have before.

$$
\begin{aligned}
& E\left\lfloor\left(\operatorname{tr}\left(\Psi B^{-1}\right)\right)^{k}\left|B^{-1}\right|^{h}\right\rfloor= \\
& 2^{k}((m p / 2)+h p)_{\{k\}} \frac{\Gamma_{p}(m / 2+h)}{2^{-h p} \Gamma_{p}(m / 2)}|\Psi|^{-h}
\end{aligned}
$$

10. $E\left\lfloor\left(\operatorname{tr}\left(\Psi B^{-1}\right)\right)^{-k}\left|B^{-1}\right|^{h}\right\rfloor=$

$$
\frac{\Gamma_{p}(m / 2+h)}{2^{h p+k}(m p / 2-k+p h)_{k} \Gamma_{p}(m / 2)}|\Psi|^{-h}
$$

\section{Proof.}

$$
E\left[\left(\operatorname{tr}\left(\Psi B^{-1}\right)\right)^{0}\left|B^{-1}\right|^{h}\right]=E\left[\left(\operatorname{tr}\left(\Psi B^{-1}\right)\right) h_{1}(B)\right]
$$

where $\quad h_{1}(B)=\left(\operatorname{tr}\left(\Psi B^{-1}\right)\right)^{-1}\left|B^{-1}\right|^{h} . \quad$ Then

$$
h_{1}(x B)=\left(\operatorname{tr}\left(\Psi(x B)^{-1}\right)\right)^{-1}\left|(x B)^{-1}\right|^{h}=x^{1-p h} h_{1}(B) \quad \text { so }
$$

that $\quad l=1-p h \quad$ and

$$
E\left[\left(\operatorname{tr}\left(\Psi B^{-1}\right)\right)^{0} h_{1}(B)\right]=[m p-2(1-p h)] E\left[h_{1}(B)\right],
$$

i.e.,

$$
\begin{aligned}
& E\left[\operatorname{tr}\left(\Psi B^{-1}\right)^{0}\left|B^{-1}\right|^{h}\right]= \\
& {[m p-2(1-p h)] E\left[\left(\operatorname{tr}\left(\Psi B^{-1}\right)\right)^{-1}\left|B^{-1}\right|^{h}\right]}
\end{aligned} .
$$


Let

$$
E\left[\left(\operatorname{tr}\left(\Psi B^{-1}\right)\right)^{-1}\left|B^{-1}\right|^{h}\right]=E\left[\left(\operatorname{tr}\left(\Psi B^{-1}\right)\right) h_{2}(B)\right]
$$

where $\quad h_{2}(B)=\left(\operatorname{tr}\left(\Psi B^{-1}\right)\right)^{-2}\left|B^{-1}\right|^{h}$. Then

$h_{2}(x B)=\left(\operatorname{tr}\left(\Psi(x B)^{-1}\right)\right)^{-2}\left|(x B)^{-1}\right|^{h}=x^{2-p h} h_{2}(B)$

so that $l=2-p h \quad$ and

$E\left[\left(t r\left(\Psi B^{-1}\right)\right)^{-1} h_{2}(B)\right]=[m p-2(2-p h)] E\left[h_{2}(B)\right]$

, i.e.,

$E\left[\operatorname{tr}\left(\Psi B^{-1}\right)^{-1}\left|B^{-1}\right|^{h}\right]=$

$[m p-2(2-p h)] E\left[\left(\operatorname{tr}\left(\Psi B^{-1}\right)\right)^{-2}\left|B^{-1}\right|^{h}\right]^{\cdot}$

Similarly, we have

$$
\begin{aligned}
& E\left[\operatorname{tr}\left(\Psi B^{-1}\right)^{0}\left|B^{-1}\right|^{h}\right]= \\
& {[m p-2(1-p h)] E\left[\left(\operatorname{tr}\left(\Psi B^{-1}\right)\right)^{-1}\left|B^{-1}\right|^{h}\right]} \\
& E\left[\operatorname{tr}\left(\Psi B^{-1}\right)^{-1}\left|B^{-1}\right|^{h}\right]= \\
& {[m p-2(2-p h)] E\left[\left(\operatorname{tr}\left(\Psi B^{-1}\right)\right)^{-2}\left|B^{-1}\right|^{h}\right]} \\
& .-.-.-. \\
& E\left[\operatorname{tr}\left(\Psi B^{-1}\right)^{-(k-2)}\left|B^{-1}\right|^{h}\right]= \\
& {[m p-2(k-1-p h)] E\left[\left(\operatorname{tr}\left(\Psi B^{-1}\right)\right)^{-(k-1)}\left|B^{-1}\right|^{h}\right]}
\end{aligned}
$$

and

$$
\begin{aligned}
& E\left[\operatorname{tr}\left(\Psi B^{-1}\right)^{-(k-1)}\left|B^{-1}\right|^{h}\right]= \\
& {[m p-2(k-p h)] E\left[\left(\operatorname{tr}\left(\Psi B^{-1}\right)\right)^{-k}\left|B^{-1}\right|^{h}\right]}
\end{aligned}
$$

That is

$$
\begin{aligned}
& \frac{1}{[m p-2(1-p h)]} E\left[\operatorname{tr}\left(\Psi B^{-1}\right)^{0}\left|B^{-1}\right|^{h}\right]= \\
& E\left[\left(\operatorname{tr}\left(\Psi B^{-1}\right)\right)^{-1}\left|B^{-1}\right|^{h}\right],
\end{aligned}
$$

$$
\begin{aligned}
& \frac{1}{[m p-2(2-p h)]} E\left[\operatorname{tr}\left(\Psi B^{-1}\right)^{-1}\left|B^{-1}\right|^{h}\right]= \\
& E\left[\left(\operatorname{tr}\left(\Psi B^{-1}\right)\right)^{-2}\left|B^{-1}\right|^{h}\right] \\
& \text {-..-.-. } \\
& \frac{1}{[m p-2(k-1-p h)]} E\left[\operatorname{tr}\left(\Psi B^{-1}\right)^{-(k-2)}\left|B^{-1}\right|^{h}\right] \\
& =E\left[\left(\operatorname{tr}\left(\Psi B^{-1}\right)\right)^{-(k-1)}\left|B^{-1}\right|^{h}\right]
\end{aligned}
$$

and

$$
\begin{aligned}
& \frac{1}{[m p-2(k-p h)]} E\left[\operatorname{tr}\left(\Psi B^{-1}\right)^{-(k-1)}\left|B^{-1}\right|^{h}\right]= \\
& E\left[\left(\operatorname{tr}\left(\Psi B^{-1}\right)\right)^{-k}\left|B^{-1}\right|^{h}\right]
\end{aligned}
$$

Finally, we have

$$
\begin{aligned}
& E\left\lfloor\left(\operatorname{tr}\left(\Psi B^{-1}\right)\right)^{-k}\left|B^{-1}\right|^{h}\right\rfloor \\
& =\frac{1}{[m p-2(k-p h)]} \times \frac{1}{[m p-2(k-1-p h)]} \times \cdots \\
& \times \frac{1}{[m p-2(2-p h)]} \times \frac{1}{[m p-2(1-p h)]} \\
& E\left[\operatorname{tr}\left(\Psi B^{-1}\right)^{0}\left|B^{-1}\right|^{h}\right] \\
& =2^{-k} \frac{1}{\frac{m p}{2}+(p h-k)} \frac{1}{\frac{m p}{2}+(p h-k+1)} \cdots \\
& \cdots \frac{1}{\frac{m p}{2}+(p h-2)} \frac{1}{\frac{m p}{2}+(p h-1)} E\left[\left|B^{-1}\right|^{h}\right] \\
& E\left\lfloor\left(\operatorname{tr}\left(\Psi B^{-1}\right)\right)^{-k}\left|B^{-1}\right|^{h}\right\rfloor \\
& =2^{-k} \times \frac{1}{\frac{m p}{2}+(p h-k)} \times \frac{1}{\frac{m p}{2}+(p h-k+1)} \times \cdots \\
& \times \frac{1}{\frac{m p}{2}+(p h-2)} \times \frac{1}{\frac{m p}{2}+(p h-1)} E\left(|B|^{-h}\right)
\end{aligned}
$$

which can be evaluated as 


$$
\begin{aligned}
& E\left[\left(\operatorname{tr}\left(\Psi B^{-1}\right)\right)^{-k}\left|B^{-1}\right|^{h}\right\rfloor= \\
& \frac{\Gamma_{p}(m / 2+h)}{2^{h p+k}(m p / 2-k+p h)_{\{k\}} \Gamma_{p}(m / 2)}|\Psi|^{-h} .
\end{aligned}
$$

\section{Acknowledgement}

The first author is highly and gratefully indebted to the Prince Mohammad Bin Fahd University, Al Khobar, Saudi Arabia, for providing all necessary research facilities during the preparation of this research paper. The authors acknowledge the excellent research support provided by Kind Fahd University of Petroleum and Minerals, Saudi Arabia especially through a project \# FT 2004(23) at the university.

\section{References:}

[1] Anderson, T.W. (2003). An Introduction to Multivariate Statistical Analysis. John Wiley and Sons. New York.

[2] de Wal and Nel, D.J. (1973). On some expectations with respect to Wishart matrices. outh African Statistics Journal, 7, 61-67.

[3] Fisher, R.A. (1915). Frequency distribution of the values of the correlation coefficient in samples from an indefinitely large population. Biometrika, 10, 507-521.

[4] Ghosh, B.K. (1966). Asymptotic expansions for the moments of the distribution of correlation coefficient. Biometrika, 53, 258262.

[5] Gupta, A.K. and Nagar, D.K. (2000). Matrix Variate Distributions. Chapman and Hall, New York.

[6] Joarder, A.H. and Ali, M.M. (1997). Estimation of the scale matrix of a multivariate $t$-model under entropy loss. Metrika, 46(1), 21--32. (ISI)

[7] Joarder, A.H. (1998). Some useful Wishart expectations based on the multivariate $t$-model. Statistical Papers, 39(2), 223 - 229. (ISI)

[8] Johnson, N.L.; Kotz, S. and Balakrishnan, N. (1995). Continuous Univariate Distributions (volume 2). John Wiley and Sons, New York.
[9] Mardia, K.V., Kent, J.T. and Bibby, J.M. (1979). Multivariate Analysis. Academic Press, New York, USA.

[10] Muirhead, R.J. (1982). Aspects of Multivariate Statistical Theory. John Wiley and Sons, New York.

[11] Press, S.J. (1982). Applied Multivariate Analysis. Robert E. Krieger Pub. Co., Florida, USA.

[12] Siskind, V. (1972). Second moments of inverse Wishrat matrix elements. Biometrika, 59, 691-692.

[13] Wishart, J. (1928). The generalized product moment distribution in samples from a normal multivariate population. Biometrika, A20, 3252.

[14] Von Rosen, D (1988). Moments for the inverted Wishart distribution. Scandinavian Journal of Statistics, 15, 97-109.

[15] Von Rosen, D. (1997). On moments of the inverted Wishart distribution. Statistics, 30(3), 259-278.

[16] Sudakar Singh Chauhan, Sanjay Kumar Bit Error Probability and Capacity Analysis of Space-Time Block Codes in Spatially Correlated MIMO Weibull Fading Channels, WSEAS Transactions on Communications, Volume 13, 2014, pp 209-216 